Lobbyregister

\section{Es kommt, greift aber zu kurz}

Erfolglose Anläufe zur Einführung eines Lobbyregisters in Deutschland hatten fast schon Tradition. Dann kam die Affäre Amthor, und die Große Koalition sah sich genötigt, endlich ein Lobbyregister einzuführen und für mehr Transparenz in der Interessenvertretung zu sorgen. Das ist für sich genommen eine gute Nachricht, denn mit dem neuen Lobbyregistergesetz, das Anfang 2022 in Kraft treten wird, müssen Interessengruppen offenlegen, mit welchen Ressourcen sie Einfluss ausüben. Erstmals werden auch Lobbyagenturen und z. B. Rechtsanwaltskanzleien gezwungen, ihre Auftraggebenden und die eingesetzten finanziellen Ressourcen offenzulegen - laut Begründung aus dem Gesetzentwurf zur ersten Lesung für jeden Auftraggeber separat. Warum man dies aber nicht deutlich ins Gesetz geschrieben hat, bleibt unklar. Endlich werden nun die Akteur:innen, die so zentral für das informationelle Lobbying sind und bisher außerhalb des Radars der Öffentlichkeit agieren, stärker in den Blick der Allgemeinheit gerückt.

Positiv ist auch, dass Lobbyist:innen und Interessenvertretungen angehalten werden, ihre Auftraggebenden und das Anliegen bei jedem Treffen klar zu benennen. Dies wird in einem Verhaltenskodex festgehalten, den der Bundestag für die Interessenvertreter festlegen wird. Hoffen wir, dass so eine Kultur der Transparenz entsteht, denn kontrollieren lassen sich diese Vorgaben in der Praxis wohl kaum. Wie wichtig die öffentliche Diskussion in laufenden Gesetzgebungsverfahren - also Transparenz - ist, zeigt die Entstehungsgeschichte des Gesetzes selbst. Nachdem der erste Entwurf bekannt wurde, gab es eine breite öffentliche Allianz, die forderte, auch die Exekutive einzubeziehen. Das verabschiedete Gesetz sieht dies vor. Als Lobbyist:in nach dem Gesetz gilt, wer für sich selbst oder im Auftrag Dritter auch die Exekutive, also Bundesregierung oder Ministerialbürokratie, kontaktiert, nicht nur die Akteure des Bundestags. Um es aber deutlich zu sagen: Bei dieser Regelung geht es nicht darum, dass die Öffentlichkeit erfährt, wer mit wem Kontakt aufnimmt oder um welche Inhalte es geht, etwa indem Stellungnahmen oder Gutachten veröffentlicht werden. Dies wird weiterhin nicht passieren. Ein legislativer Fußabdruck kommt ebenso wenig wie eine Berichtspflicht, über die sich politische Kontaktaufnahmen und Netzwerke der Einflussnahme abbilden lassen. Beim Einbezug der Exekutive geht es allein um die Definition, wer als Lobbyist:in im Sinne des Gesetzes gilt und damit berichtspflichtig über

(C) Der/die Autor:in(nen) 2021. Open Access: Dieser Artikel wird unter der Creative Commons Namensnennung 4.0 International Lizenz veröffentlicht (creativecommons.org/licenses/by/4.0/deed.de).

Open Access wird durch die ZBW - Leibniz-Informationszentrum Wirtschaft gefördert. finanzielle Aufwendungen wird. Die Berichtspflicht besteht nur bei Kontaktaufnahme bis herunter zur Ebene der Unterabteilungsleiter:innen, Kontakte auf Referatsebene gelten nicht. Begründet wird dies damit, dass auf dieser Ebene die „administrative Arbeit im Mittelpunkt steht“. Als ob dort kein Einfluss ausgeübt würde.

Problematisch wird es, wenn es in die Details des Gesetzes geht. Wie im Wirtschaftsdienst vom Februar 2021 schon dargelegt, ist das Lobbyregister schlecht konzipiert, weil sein Zweck nicht deutlich wird. Transparenz ist kein Wert an sich. Beispielsweise müssen Daten nur jährlich aktualisiert werden, die Angaben insbesondere zu den personellen Aufwendungen fallen viel zu grob aus, und die Benennung der Aktivitäten kann zu lax erfolgen. Hinzu kommen die weiten Ausnahmebereiche, die von einer Registrierungspflicht befreit sind. Neben Kirchen und Religionsgemeinschaften, die generell keine Angaben machen müssen, sind dies Arbeitnehmer- und Arbeitgeberverbände, sofern sie „Einfluss auf Arbeits- und Wirtschaftsbedingungen" nehmen, sowie kommunale Spitzenverbände. Interessant ist, dass diese ohnehin schon zu weit gefassten Ausnahmebereiche noch einmal erweitert wurden. Neu umfassen sie z. B. auch Rechtsanwält:innen, wenn sie an die Allgemeinheit gerichtete Erörterungen von Rechtsfragen erbringen, auch dann, wenn dies im Auftrag Dritter geschieht. Hier denkt man unwillkürlich an Guttenberg und seine Publikationen in öffentlichen Medien, die er mutmaßlich im Auftrag als Lobbyist anfertigte. Geschieht dies in Zukunft durch einen Rechtsanwalt (und nicht durch ein Beratungsunternehmen, wie im genannten Fall), würde dies wohl nicht vom Gesetz erfasst.

Positiv ist, dass nun klarer festgelegt wurde, dass die Finanzierung von Interessengruppen offengelegt werden muss, sofern Spenden und Zuschüsse den Betrag von 20.000 Euro jährlich pro Geber überschreiten. Hier hätte man sich an den niedrigeren Grenzen des Parteiengesetzes orientieren können, die vor dem Hintergrund der Causa Spahn ohnehin kontrovers diskutiert werden. Auch ist gut, dass nun Personalkosten aus den Berichtspflichten nicht mehr herausgerechnet werden, was der ursprüngliche Entwurf noch vorsah. So bleibt insgesamt ein zwiespältiges Bild. Die Etablierung eines Lobbyregisters ist überfällig, seine Umsetzung aber halbherzig. Wenn es schlecht läuft, dienen die wenig aussagekräftigen und zu pauschalen Angaben gerade denen, die unsere öffentlichen Amtstragenden ohnehin permanent unter Generalverdacht stellen. Das ist schade, denn erstens haben sie das nicht verdient, und zweitens steckt in gut gemachter Transparenz das Potenzial zur Stärkung öffentlichen Vertrauens.

Andreas Polk Hochschule für Wirtschaft und Recht Berlin polk@hwr-berlin.de 